\title{
The Incidence, Risk Factors, Predictors, and Prognoses of Cytomegalovirus Reactivation in Immunocompetent Mechanical Ventilation Patients: A Prospective Observational Study
}

\section{Zhihui Zhang}

First Affiliated Hospital of Guangzhou Medical University

\section{Xuesong Liu}

First Affiliated Hospital of Guangzhou Medical University

\section{Ling Sang}

First Affiliated Hospital of Guangzhou Medical University

Sibei Chen

First Affiliated Hospital of Guangzhou Medical University

\section{Zhan Wu}

First Affiliated Hospital of Guangzhou Medical University

Jierong Zhang

First Affiliated Hospital of Guangzhou Medical University

\section{Yining Sun}

First Affiliated Hospital of Guangzhou Medical University

\section{Yonghao Xu}

First Affiliated Hospital of Guangzhou Medical University

\section{Weiqun He}

First Affiliated Hospital of Guangzhou Medical University

\section{Yimin Li}

First Affiliated Hospital of Guangzhou Medical University

Xiaoqing Liu ( $\sim$ lxq11118@126.com)

First Affiliated Hospital of Guangzhou Medical University

\section{Research article}

Keywords: Cytomegalovirus (CMV) reactivation, Immunocompetent, Critically ill, Epidemiology, Predictors

Posted Date: February 19th, 2021

DOI: https://doi.org/10.21203/rs.3.rs-247841/v1 
License: (c) (i) This work is licensed under a Creative Commons Attribution 4.0 International License. Read Full License 


\section{Abstract}

Background: Cytomegalovirus (CMV) reactivation can seriously affect the clinical prognoses of critically ill patients. However, the epidemiology and predictors of CMV in immunocompetent patients with mechanical ventilation are not very clear.

Methods: A single-center, prospective observational study (conducted from June 30, 2017 to July 01, 2018) with a follow-up of 90 days (September 29, 2018) that included 71 CMV-seropositive immunocompetent patients with mechanical ventilation at a 37-bed university hospital general intensive care unit (ICU) in China. Routine detection of CMV DNAemia was performed once a week until 28 days (Day $1,7,14,21,28)$ and serology, laboratory findings, and clinical data were obtained at admission.

Results: Among 71 patients, 13 (18.3\%) showed CMV reactivation within ICU 28-day admission. The median time to reactivation was 7 days. CMV reactivation was related to various factors, including body weight, body mass index (BMI), sepsis, NT-proBNP, BUN, and Hb levels $(P<0.05)$. In the multivariate regression model, $\mathrm{BMI}, \mathrm{Hb}$ level, and sepsis were associated with $\mathrm{CMV}$ reactivation patients $(P<0.05)$. Moreover, the area under the curve (AUC) of $\mathrm{BMI}, \mathrm{Hb}$ and $\mathrm{BMI}$ combined $\mathrm{Hb}$ was $0.69,0.70,0.76$, respectively. The rate of complications, duration of mechanical ventilation, hospitalization expense, length of ICU stay, and 90-day all-cause mortality rate in patients with CMV reactivation was significantly higher than in those without CMV reactivation $(P<0.05)$.

Conclusions: Among immunocompetent patients with mechanical ventilation, the incidence of CMV reactivation was high. CMV reactivation can lead to various adverse prognoses. $\mathrm{BMI}, \mathrm{Hb}$, and sepsis were independent risk factors for $\mathrm{CMV}$ reactivation. $\mathrm{BMI}$ and $\mathrm{Hb}$ may predict $\mathrm{CMV}$ reactivation.

\section{Introduction}

Cytomegalovirus (CMV) infection is widespread in the general population, and its seroprevalence is as high as $83 \%$ [1]. Primary infection is mostly during pregnancy or childhood, and the infection rate is related to several factors, such as the geographic environment and medical level [2]. CMV has the characteristics of herpes virus infection. When the body is infected with CMV, it will carry it throughout life $[2,3]$. CMV infection status is a latent infection, nevertheless, reactivation occurs under certain conditions.

The immune function is closely related to the occurrence of $\mathrm{CMV}$ reactivation. CMV can reactivate in the course of diminished immunity and frailty $[3,4]$. Critically ill patients have severe diseases (such as sepsis, burns, and ARDS) and impaired immune function [5-7]. CMV is prone to reactivation in ICU patients. In recent decades, numerous studies conducted in developed countries have suggested that the incidence of CMV reactivation in critically ill immunocompetent patients is $9-71 \%$ [8]. Moreover, CMV reactivation can lead to various adverse prognoses, such as prolonged mechanical ventilation \& ECMO duration, increased length of hospitalization and mortality [7-9]. However, CMV reactivation is challenging to predict earlier because it lacks particular clinical manifestations. At present, CMV reactivation is mainly diagnosed by measuring the viral load $[2,10]$. Some studies have shown that CMV 
reactivation may be associated with sepsis, transfusion, and cytokine levels (IL, TNF). Still, none of them clearly screened out effective indicators to predict CMV reactivation $[4,5,8,11]$.

Therefore, there is an urgent need to find indicators that can effectively predict CMV reactivation in critically ill immunocompetent patients and to study the epidemiology of CMV reactivation in critically ill patients in developing countries.

\section{Methods}

\subsection{Patient selection}

This study was conducted in the general ICU of the First Affiliated Hospital of Guangzhou Medical University, a national teaching hospital with 2000 beds; the ICU has 37 independent beds. During a 1-year research period (June 30, 2017 to July 01, 2018), all patients who met the study criteria were incorporated into the research group. The research group was recorded and detected for relevant indicators in the set time point. The study was given official approval by the Ethics Committee of the First Affiliated Hospital of Guangzhou Medical University and authorized by the Chinese Clinical Trial Registry. The patient's consent (or that of their family members) through signed informed consent forms.

\subsection{Exclusion criteria}

(1) Inability to provide informed consent; (2) Age < 18 years; (3) Pregnant or lactation; (4) Survival time < 72 h; (5) Readmitted to ICU; (6) CMV seronegative; (7) Invasive mechanical ventilation before admission; (8) Received antiviral drugs before admission; (9) Diagnosed with solid organ or bone marrow tumor; (10) Neutropenic (white cell counts $<1000 / \mathrm{uL}$ or neutrophils $<500 / \mathrm{uL}$ ); (11) Systemic glucocorticoids were used (prednisone $>0.1 \mathrm{mg} / \mathrm{kg}$ for $>3$ months, methylprednisolone $>40 \mathrm{mg} / \mathrm{d}$ for $>1$ week, or equivalent); (12) Diagnosed immunodeficiency (transplantation, HIV or immunosuppressive drugs).

\subsection{Study design}

Screening of all patients admitted to ICU from June 30, 2017 to July 01, 2018. Patients who met the exclusion criteria were excluded from the study. Data are regularly recorded until the subject is discharged from ICU or general ward (or death). On September 29, 2018, the study ended (a follow-up of 90 days). The subjects were divided into two groups based on whether CMV DNAemia was more than 500 copies $/ \mathrm{mL}$, the reactivation and non-reactivation groups. Routine detection of CMV DNAemia once a week until 28 days (Day 1, 7, 14, 21, 28), and serology, laboratory findings, and clinical data were obtained at admission.

\subsection{Procedures and data collection}

Two trained researchers performed CMV DNAemia testing, and the results were recorded on electronic records. The clinical data from 71 cases, including patient demographics, clinical symptoms and signs, laboratory findings, and clinical outcomes, were extracted from the electronic records by two independent 
intensivists who subsequently cross-checked the data for accuracy. A third independent reviewer resolved the disagreement. All data were entered into the computerized database for further statistical analyses.

\subsection{Study definitions}

CMV serology was determined in a plasma sample obtained $24 \mathrm{~h}$ within admission using an enzyme immunoassay, which was performed according to the manufacturer's instructions (Human AntiCytomegalovirus IgG, Abcam Products, Cambridge, United Kingdom).

Subsequently, a real-time Taqman CMV DNA polymerase chain reaction was used to determine the viral load in plasma for seropositive patients. The viral load was detected once a week for 28 days (Day 1, 7, $14,21,28)$. Viral load values were calibrated to the CMV World Health Organization Standard. CMV reactivation was defined as a load greater than or equal to 500 copies/mL. Screening for CMV serology or viral load was part of routine clinical practice in this hospital. Both serology and reactivation results from this study were made available to the treating physicians. However, it was up to the attending physician to decide whether the patient should be treated.

\subsection{Statistical analysis}

Continuous variables were expressed as mean \pm SD or median (interquartile ranges, IQRs) and compared with the Wilcoxon rank-sum test. Categorical variables were expressed as counts and percentages, and compared using the chi-square test (Fisher's exact test) as appropriate. The risk factors for CMV reactivation were screened using a univariate logistic regression model. Variables with a P-value of 0.05 or less were considered the potential risk factors and further imported into the multivariate logistic regression analysis. The $\mathrm{CMV}$ reactivation risk model was established by calculating the regression coefficient $(\beta)$, odds ratio (OR), and 95\% confidence interval $(\mathrm{Cl})$. The receiver operating curve (ROC) was used to evaluate the predictive value of CMV reactivation. The AUC, 95\% Cl, P-value, cut off, sensitivity, and specificity were calculated. Kaplan-Meier (KM) survival analysis was used to compare the 90-day survival rate between the two groups, and the log-rank test was used to compare the survival curve and Hazard Ratio (HR). The significance threshold was set at a 2-sided P value of less than 0.05. All statistical analyses or charting were performed using SPSS version 25.0 (SPSS Inc., USA) and GraphPad Prism 8.0 (GraphpadSoftware Inc., USA).

\section{Results}

\subsection{Incidence of CMV reactivation}

A total of 1,350 patients were admitted to ICU during the study period. As shown in Fig. 1, 91 patients were initially screened for inclusion in the study, and 20 were excluded because of solid organ tumors (n = 9), IMV less than $24 \mathrm{~h}(\mathrm{n}=4)$, sputum smears positive after admission $(n=3)$, death or discharged within $72 \mathrm{~h}$ of admission ( $n=3)$, and HIV ( $n=1)$. Eventually, 71 patients were enrolled. 
Sensationally, CMV seropositivity was observed to be $100 \%$ during patients screening. Among the 71 enrolled patients, there were 13 cases of CMV reactivation within a 28-day ICU entry, corresponding to an incidence of CMV reactivation of 18.3\% (shown in Fig. 2). Of 13 patients with CMV reactivation, 6 (46.1\%) were reactivated within 24 hours after entering ICU, 1 (7.7\%) on Day-7, $4(30.8 \%)$ on Day-14, 1 (7.7\%) on Day-21 and 1 (7.7\%) on Day-28 (shown in Fig. 3). CMV DNAemia was also found to be severe: 8 (61.5\%) reached 2001-3000 copies/mL, 3 (23.1\%)reached 3001-4000 copies/mL and 2 (15.4\%) reached > 5000 copies/mL, shown in Fig. 4.

\subsection{Patients' characteristics}

The patients' characteristics of the study population at ICU admission are shown in Table 1. We included 71 patients with complete data, of whom 58 were men (81.7\%). The mean age was 64 years. The data were revealing in two ways. The single most striking observation to emerge from the data comparison was that patients with $\mathrm{CMV}$ reactivation had lower body weight [mean: $51.1 \pm 10.6 \mathrm{vs} .61 .6 \pm 11.6(\mathrm{~kg}), \mathrm{P}$ $=0.014$ ] and BMI [mean: $19.9 \pm 4.1$ vs. $22.1 \pm 3.4\left(\mathrm{~kg} / \mathrm{m}^{2}\right), \mathrm{P}=0.031$ ] than controls. There were also differences in sepsis of between CMV reactivation and non-CMV reactivation [53.8\% vs. $19.0 \%(\mathrm{n}), \mathrm{P}=$ $0.031]$. There were, however, no significant differences in other clinical characteristics between CMV reactivation and controls. 
Table 1

Clinical characteristics of the study patients at ICU admission.

\begin{tabular}{|c|c|c|c|c|}
\hline & Overall & CMV reactivatio & & \\
\hline & $N=71$ & Yes & No & $\mathrm{P}$ \\
\hline & [Mean, n (\%)] & $(n=13,18.3 \%)$ & $(n=58,81.7 \%)$ & \\
\hline Age (yrs) & 64 & $68 \pm 12$ & $63 \pm 14$ & 0.20 \\
\hline Gender, n (\%) & & & & 0.24 \\
\hline Male & $58(81.7)$ & $9(69.2)$ & 49 (84.5) & - \\
\hline Female & $13(18.3)$ & $4(30.8)$ & $9(15.5)$ & - \\
\hline Height (cm) & 165 & $160(10)$ & $170(12)$ & 0.10 \\
\hline Weight $(\mathbf{k g})^{a}$ & 58.5 & $51.1 \pm 10.6$ & $61.6 \pm 11.6$ & 0.014 \\
\hline $\mathrm{BMI}\left(\mathrm{kg} / \mathrm{m}^{2}\right)^{\mathrm{a}}$ & 21.3 & $19.9 \pm 4.1$ & $22.1 \pm 3.4$ & 0.031 \\
\hline \multicolumn{5}{|l|}{ Severity of Score } \\
\hline APACHE $\square$ & 20 & $22 \pm 8$ & $20 \pm 7$ & 0.37 \\
\hline SOFA & 8 & $8(7)$ & $8(5)$ & 0.80 \\
\hline \multicolumn{5}{|l|}{ Comorbidities, n(\%) } \\
\hline Hypertension & $34(47.9)$ & $6(46.2)$ & $28(48.3)$ & NA \\
\hline COPD & $28(39.4)$ & $6(46.2)$ & $22(37.9)$ & 0.76 \\
\hline Coronary Heart Disease & $19(26.8)$ & $4(30.8)$ & 15 (25.9) & 0.74 \\
\hline Diabetes & $18(25.4)$ & $2(15.4)$ & $16(27.6)$ & 0.49 \\
\hline Chronic Kidney Disease & $6(8.5 \%)$ & $1(7.7)$ & $5(8.6)$ & NA \\
\hline Bronchiectasis & $8(11.3)$ & $1(7.7)$ & $7(12.1)$ & NA \\
\hline Asthma & $2(2.8)$ & $1(7.7)$ & $1(1.7)$ & 0.34 \\
\hline Rheumatic Heart Disease & $1(1.4)$ & $0(0)$ & $1(1.7)$ & NA \\
\hline \multicolumn{5}{|l|}{ Complications, $n(\%)$} \\
\hline Surgical Factors & $14(19.7)$ & $1(7.7)$ & $13(22.4)$ & 0.44 \\
\hline Heart Surgery & $13(18.3)$ & $1(7.7)$ & $12(20.7)$ & 0.44 \\
\hline
\end{tabular}

a, $\mathrm{P}<0.05$; Continuous variables were expressed as Mean $\pm \mathrm{SD}$ or Median (IQRs); Data in bold indicated the difference was statistically significant. 


\begin{tabular}{|c|c|c|c|c|}
\hline & Overall & $\mathrm{CMV}$ react & & \\
\hline Trauma & $1(1.4)$ & $0(0)$ & $1(1.7)$ & NA \\
\hline Internal Medicine Factors & $57(80.3)$ & $12(92.3)$ & $45(77.6)$ & 0.44 \\
\hline Severe Pneumonia & $48(67.6)$ & $10(76.9)$ & $38(65.5)$ & 0.53 \\
\hline AECOPD & $25(35.2)$ & $5(38.5)$ & $20(34.5)$ & 0.76 \\
\hline Sepsis $^{a}$ & $18(25.4)$ & $7(53.8)$ & $11(19.0)$ & 0.015 \\
\hline AKI & $17(23.9)$ & $4(30.8)$ & $13(22.4)$ & 0.50 \\
\hline Bronchiectasis Infection & $8(11.3)$ & $1(7.7)$ & $7(12.1)$ & NA \\
\hline ARDS & $7(9.9)$ & $3(23.1)$ & $4(6.9)$ & 0.11 \\
\hline Acute Asthma Attack & $3(4.2)$ & $1(7.7)$ & $2(3.4)$ & 0.46 \\
\hline Acute Coronary Syndrome & $3(4.2)$ & $0(0)$ & $3(5.2)$ & NA \\
\hline Heart Valve Disease & $2(2.8)$ & $0(0)$ & $2(3.4)$ & NA \\
\hline Liver Failure & $1(1.4)$ & $0(0)$ & $1(1.7)$ & NA \\
\hline Acute Suppurative Cholangitis & $1(1.4)$ & $0(0)$ & $1(1.7)$ & NA \\
\hline
\end{tabular}

We next focused on the patients' clinical indicators within a 24-hour ICU admission. From the data in Table 2 and Table 3, patients with CMV reactivation had markedly higher levels of NT-proBNP [median: 7745 vs. 1805 (pg/mL), $P=0.014$ ], BUN [median: 13.6 vs. 8.6 (mmol/L), $P=0.03)$ ]and Hemoglobin [median: 98.0 vs. 104.0 (g/L), $P=0.029$ ]. Nevertheless, there were no statistically evident differences for immune indicators between the two groups. 
Table 2

Clinical indicators and laboratory findings of the study patients within 24-hour ICU admission.

Overall

$N=71$

[Mean, $\mathrm{n}(\%)$ ]
CMV reactivation

Yes

$(n=13,18.3 \%) \quad(n=58,81.7 \%)$

\section{Basic Vital Signs}

$\begin{array}{lllll}\text { Average Blood Pressure }(\mathrm{mmHg}) & 91.5 & 95(23) & 92(23) & 0.36 \\ \text { Heart Rate }(\mathrm{bp}) & 110 & 115 \pm 26 & 109 \pm 27 & 0.47 \\ \text { Respiratory Rate }(\mathrm{t} / \mathrm{m}) & 24 & 26(7.5) & 23(6) & 0.89 \\ \text { Temperature }\left({ }^{\circ} \mathrm{C}\right) & 37.1 & 36.6(0.75) & 36.9(0.6) & 0.43 \\ \mathrm{SPO}_{2}(\%) & 94 & 97(8) & 97(6) & 0.88\end{array}$

\section{Laboratory Findings}

$\mathrm{P} / \mathrm{F}$

Nt-proBNP $(p g / m L)^{a}$

AST (U/L)

$\operatorname{ALT}(\mathrm{U} / \mathrm{L})$

T-BIL $(\mu \mathrm{mol} / \mathrm{L})$

$\operatorname{Scr}(\mu \mathrm{mol} / \mathrm{L})$

BUN (mmol/L)

PT (s)

$\operatorname{APTT}(s)$

PCT $(\mathrm{ng} / \mathrm{mL})$

Hypersensitive CRP (mg/L)

$\operatorname{ESR}(\mathrm{mm} / \mathrm{h})$

G Test $(\mathrm{pg} / \mathrm{mL})$

GM Test (Aspergillus) ( $\mu \mathrm{g} / \mathrm{L})$

GM Test (Cryptococcus) ( $\mu \mathrm{g} / \mathrm{L})$
234

7267

176.6

97.1

21.7

191.7

11.9

16.8

44.6

10.0

97.8

54.6

77.0

0.31

$5(7.0)$
$217 \pm 76$

7745 (18929)

41.0 (76.7)

26.5 (49.7)

10.9 (9.1)

133 (155.5)

13.6 (10.5)

15.7 (3.4)

43 (16.4)

$2.5(11.5)$

$107.2(81.3)$

42 (66.5)

21.11 (68.49)

$0.32(0.27)$

$0(0)$
$238 \pm 97$

0.49

1805 (7094)

42.8 (53.8)

24.4 (36.0)

$15.2(29.6)$

128 (160.8)

8.6 (8.7)

0.03

$5.6(2.6)$

0.82

40.5 (11.3)

0.67

0.9 (7.2)

0.36

98.4 (116.3)

0.34

45 (67)

0.81

$10.43(45.88)$

0.50

$0.36(0.44)$

0.71

5 (8.6)

0.58

a, $\mathrm{P}<0.05 ; \mathrm{PaO}_{2} / \mathrm{FiO}_{2}$ ratio $(\mathrm{P} / \mathrm{F})$ : the ratio between the arterial partial pressure of oxygen and the inspiratory concentration of oxygen; Continuous variables were expressed as Mean \pm SD or Median (IQRs); Data in bold indicated the difference was statistically significant. 


\begin{tabular}{|c|c|c|c|c|}
\hline & Overall & \multicolumn{3}{|c|}{ CMV reactivation } \\
\hline White Blood Cells $\left(10^{9} / \mathrm{L}\right)$ & 14.2 & $14.8(11.7)$ & $12.4(7.4)$ & 0.28 \\
\hline Neutrophils $\left(10^{9} / \mathrm{L}\right)$ & 12.8 & $13.3(11.3)$ & $11.1(7.5)$ & 0.22 \\
\hline Lymphocytes $\left(10^{9} / \mathrm{L}\right)$ & 0.6 & $0.4(0.4)$ & $0.5(0.6)$ & 0.19 \\
\hline Monocytes $\left(10^{9} / \mathrm{L}\right)$ & 0.7 & $0.4(0.8)$ & $0.7(0.6)$ & 0.16 \\
\hline Eosinophils $\left(10^{9} / \mathrm{L}\right)$ & 0.07 & $0(0.16)$ & $0(0.01)$ & 0.32 \\
\hline Basophils $\left(10^{9} / \mathrm{L}\right)$ & 0.03 & $0(0.07)$ & $0(0.01)$ & 0.33 \\
\hline Erythrocyte $\left(10^{12} / \mathrm{L}\right)$ & 3.40 & $3.05 \pm 0.79$ & $3.52 \pm 0.84$ & 0.07 \\
\hline Hemoglobin $(g / L)^{a}$ & 99.9 & $98.0(35.0)$ & $104(34.3)$ & 0.029 \\
\hline Platelet $\left(10^{9} / \mathrm{L}\right)$ & 177 & $190 \pm 116$ & $174 \pm 86$ & 0.65 \\
\hline \multicolumn{5}{|c|}{$\begin{array}{l}\text { a, } \mathrm{P}<0.05 ; \mathrm{PaO}_{2} / \mathrm{FiO}_{2} \text { ratio }(\mathrm{P} / \mathrm{F}) \text { : the ratio between the arterial partial pressure of oxygen and the } \\
\text { inspiratory concentration of oxygen; Continuous variables were expressed as Mean } \pm \mathrm{SD} \text { or Median } \\
\text { (IQRs); Data in bold indicated the difference was statistically significant. }\end{array}$} \\
\hline
\end{tabular}


Table 3

Immune indicators of the study patients within 24-hour ICU admission.

\begin{tabular}{|c|c|c|c|c|}
\hline & Overall & CMV reactivatic & & \\
\hline & $N=71$ & Yes & No & $\mathrm{P}$ \\
\hline & [Mean] & $(n=13,18.3 \%)$ & $(n=58,81.7 \%)$ & \\
\hline \multicolumn{5}{|l|}{ Lymphocyte Subpopulations (\%) } \\
\hline T Lymphocytes & 64.4 & $63.1 \pm 17.1$ & $64.7 \pm 11.7$ & 0.74 \\
\hline T-helper Lymphocytes (Th) & 39.2 & $39.7 \pm 13.8$ & $39.1 \pm 11.0$ & 0.88 \\
\hline T-suppressor Lymphocytes (Ts) & 22.2 & $19.9(12.8)$ & $19.5(9.0)$ & 0.90 \\
\hline Th/Ts & 2.2 & $1.9(1.8)$ & $1.9(1.1)$ & 0.99 \\
\hline B Lymphocytes & 19.2 & $24.5 \pm 16.8$ & $18.0 \pm 10.2$ & 0.21 \\
\hline NK & 13.9 & $10.3 \pm 6.7$ & $14.7 \pm 10.7$ & 0.07 \\
\hline \multicolumn{5}{|l|}{ Immune Molecules (g/L) } \\
\hline $\lg G$ & 10.8 & $8.5(4.5)$ & $9.9(4.5)$ & 0.18 \\
\hline $\lg A$ & 2.3 & $1.4(1.5)$ & $1.9(1.7)$ & 0.15 \\
\hline $\lg M$ & 0.9 & $0.6(0.3)$ & $0.7(0.4)$ & 0.20 \\
\hline C3 & 0.7 & $0.7(0.3)$ & $0.7(0.3)$ & 0.99 \\
\hline C4 & 0.2 & $0.2 \pm 0.1$ & $0.2 \pm 0.1$ & 0.58 \\
\hline \multicolumn{5}{|l|}{ Th1/Th2 Cytokines (mmol/L) } \\
\hline IL-2 & 10.4 & $10.5(6.9)$ & $10.3(6.3)$ & 0.77 \\
\hline IL-4 & 2.2 & $2.2(1.4)$ & $2.3(1.1)$ & 0.60 \\
\hline IL-6 & 11.2 & $5.4(3.9)$ & $5.1(4.3)$ & 0.75 \\
\hline IL-8 & 41.1 & $42.2(52.1)$ & $26.0(25.4)$ & 0.30 \\
\hline IFN- $\gamma$ & 3.0 & $2.9(0.9)$ & $2.5(1.9)$ & 0.98 \\
\hline TNF-a & 44.2 & $47.8(35.5)$ & $39.4(19.1)$ & 0.30 \\
\hline GM-CSF & 2.7 & $2.6(0.2)$ & $2.6(0.6)$ & 0.56 \\
\hline
\end{tabular}

Overall, these results indicated that CMV reactivation was associated with body weight, BMI, sepsis, NTproBNP, BUN, and $\mathrm{Hb}$ levels.

\subsection{Risk factors and predictors}


Multivariable logistic regression analysis of factors associated with CMV reactivation is shown in Table 4. In the multivariate regression model, BMI level [OR: $1.25,95 \% \mathrm{Cl}: 1.03-1.53, \mathrm{P}=0.026$ ], Hb level [OR:1.04, 95\% Cl: $1.01-1.08, \mathrm{P}=0.026$ ] and sepsis [OR: $0.10,95 \% \mathrm{Cl}: 0.02-0.50, \mathrm{P}=0.005]$ were associated with patients with $C M V$ reactivation. Based on the regression coefficient ( $\beta$ ), BMI [B: -0.23$]$ and $\mathrm{Hb}[\beta$ : -0.04] were protective factors, while sepsis [ $\beta$ : 2.32] was a risk factor. Meanwhile, these results indicated that the risk of CMV reactivation increased by $125 \%$ for each $1 \mathrm{~kg} / \mathrm{m}^{2}$ decrease in BMI level, increased by $104 \%$ for each $1 \mathrm{~g} / \mathrm{L}$ decrease of $\mathrm{Hb}$ level, and increased by $10 \%$ once sepsis occurred.

Table 4

Risk factors associated with CMV reactivation in multivariate regression analysis.

\begin{tabular}{|llllll|}
\hline Variables & $\boldsymbol{\beta}$ & Wald & OR & $95 \% \mathrm{Cl}$ & $\boldsymbol{P}$ \\
\hline $\mathrm{BMI}\left(\mathrm{kg} / \mathrm{m}^{2}\right)^{\mathrm{a}}$ & -0.23 & 4.95 & 1.25 & $1.03-1.53$ & 0.026 \\
\hline $\mathrm{Hb}(\mathrm{g} / \mathrm{L})^{\mathrm{a}}$ & -0.04 & 4.99 & 1.04 & $1.01-1.08$ & 0.026 \\
\hline Sepsis $^{\mathrm{b}}$ & 2.32 & 7.74 & 0.10 & $0.02-0.50$ & 0.005 \\
\hline a $, \mathrm{P}<0.05{ }^{\text {b }}{ }^{\mathrm{b}}, \mathrm{P}<0.01 ; \beta$ : Regression Coefficient; OR: Odds Ratio; Cl: $95 \%$ Confidence Interval. \\
\hline
\end{tabular}

Furthermore, we plotted ROC curves for BMI and $\mathrm{Hb}$ levels to assess CMV reactivation's predictive value. From Table 5 and Fig. 5 , it could be seen that the AUC of BMI was 0.69 [Specificity (\%): 72.4, Sensitivity (\%): $69.2 ; 95 \% \mathrm{Cl}: 0.51-0.87 ; \mathrm{P}=0.034$ ], $\mathrm{Hb}$ was 0.70 [Specificity (\%): 48.3 , Sensitivity (\%): $100 ; 95 \% \mathrm{Cl}$ : 0.57-0.83; $\mathrm{P}=0.023$ ], and BMI combined $\mathrm{Hb}$ was 0.76 [Specificity (\%): 70.7, Sensitivity (\%): $76.9 ; 95 \% \mathrm{Cl}$ : $0.60-0.91 ; \mathrm{P}=0.004]$. In summary, these AUC results showed that $\mathrm{BMI}$ and $\mathrm{Hb}$ had a moderate predictive value for CMV reactivation. We also used the cut-off method to obtain $\mathrm{BMI}<22.3 \mathrm{~kg} / \mathrm{m}^{2}$ as the threshold for predicting $\mathrm{CMV}$ reactivation, and $\mathrm{Hb}<87 \mathrm{~g} / \mathrm{L}$ as the threshold for predicting $\mathrm{CMV}$ reactivation.

Table 5

Predictive value of $\mathrm{BMI}$ and $\mathrm{Hb}$ on $\mathrm{CMV}$ reactivation evaluated by ROC.

\begin{tabular}{|c|c|c|c|c|c|c|}
\hline & AUC & Cut off & Specificity (\%) & Sensitivity (\%) & $95 \% \mathrm{Cl}$ & $P$ \\
\hline BMI $\left(\mathrm{kg} / \mathrm{m}^{2}\right)^{\mathrm{a}}$ & 0.69 & 22.3 & 72.4 & 69.2 & $0.51-0.87$ & 0.034 \\
\hline $\mathrm{Hb}(\mathrm{g} / \mathrm{L})^{\mathrm{a}}$ & 0.70 & 87 & 48.3 & 100 & $0.57-0.83$ & 0.023 \\
\hline BMl combined $\mathrm{Hb}^{\mathrm{b}}$ & 0.76 & - & 70.7 & 76.9 & $0.60-0.91$ & 0.004 \\
\hline
\end{tabular}

\subsection{Clinical prognoses}

It is clear from Table 6 that of 71 patients, 20 deceased within 90 days of ICU admission [90-day all-cause mortality: $28.2 \%]$, and non-survivors were more likely to be $\mathrm{CMV}$ reactivation cases compared with $\mathrm{CMV}$ 
non-reactivation cases [69.2\% vs. $19.0 \%$ (n), $P=0.001]$. As Fig. 6 shows, the Kaplan-Meier Curve to evaluate patients' 90-day survival was a significant difference [95\% Cl: 49.54-72.46, Log rank < 0.01] between the two groups. No evidence was found for 28-day mortality associations between CMV reactivation cases and controls.

Table 6

Clinical prognoses of the study patients with and without CMV reactivation.

\begin{tabular}{|c|c|c|c|c|}
\hline & Overall & $\mathrm{CMV}$ reactiv & & \\
\hline & $N=71$ & Yes & No & $P$ \\
\hline & $\begin{array}{l}\text { [Mean, n } \\
\text { (\%)] }\end{array}$ & $\begin{array}{l}(n=13, \\
18.3 \%)\end{array}$ & $\begin{array}{l}(n=58, \\
81.7 \%)\end{array}$ & \\
\hline Complications, $\mathrm{n}(\%)^{\text {\&a }}$ & $54(76.1)$ & $13(100)$ & $41(70.7)$ & 0.029 \\
\hline Blood Transfusion in ICU, n (\%) & $52(73.2)$ & $12(92.3)$ & $40(69.0)$ & 0.16 \\
\hline $\begin{array}{l}\text { Length of Invasive Mechanical Ventilation } \\
\text { (d) })^{\mathrm{b}}\end{array}$ & 27 & $25(30)$ & $10(10)$ & 0.005 \\
\hline Hospitalization Expenses (million/ $¥)^{a}$ & 0.25 & $0.35(0.43)$ & $0.16(0.12)$ & 0.023 \\
\hline Length of Hospital Stay (d) & 42 & $50(40)$ & $28(22)$ & 0.10 \\
\hline ICU Length of Stay(d) ${ }^{b}$ & 27 & $27(30)$ & $12(10)$ & 0.004 \\
\hline 28-day All-cause Mortality, n (\%) & $17(23.9)$ & $5(38.5)$ & $12(20.7)$ & 0.28 \\
\hline 90-day All-cause Mortality, n (\%) ${ }^{\mathrm{b}}$ & $20(28.2)$ & $9(69.2)$ & $11(19.0)$ & 0.001 \\
\hline
\end{tabular}

Significantly more CMV reactivation cases had length of invasive mechanical ventilation [median: 25 vs. 10 (days), $P=0.005$ ], hospitalization expense[median: 0.35 vs. 0.16 (million/ $¥), P=0.023$ ] and length of ICU stay [median: 27 vs. 12 (days), $P=0.004$ ]. The complications rate $[100 \%$ vs. $70.7 \%(n), P=0.029$ ] was initiated significantly more frequently in $\mathrm{CMV}$ reactivation patients than in controls. No significant differences were found between the rate of blood transfusion and length of hospital stay. Therefore, CMV reactivation can lead to various adverse clinical outcomes.

\section{Discussion}

This study sought to investigate the incidence, risk factors, predictors, and prognosis of CMV reactivation in immunocompetent mechanical ventilation patients. We have found that, among immunocompetent patients with mechanical ventilation, the CMV reactivation incidence was $18.3 \%$. Clinical features, including body weight, BMI, sepsis, and biochemical indicators (NT-proBNP, BUN, Hb level) were 
associated with the development of $\mathrm{CMV}$ reactivation. Further statistical analysis showed that $\mathrm{BMI}, \mathrm{Hb}$, and sepsis had predictive value and were independent risk factors for CMV reactivation. In addition, CMV reactivation could lead to several adverse outcomes.

CMV reactivation has been a frequent phenomenon among patients admitted to the ICU. A 1990-2016 systematic review and meta-analysis review that included cohort studies estimated the incidence of CMV reactivation to be $31 \%$ in immunocompetent patients $(95 \% \mathrm{Cl}, 24-39 \%)$ in critical care settings [12]. Similarly, a recent study that enrolled 55 patients with non-immunosuppressed cirrhosis demonstrated that the cumulative incidence of active CMV infection was 30.9\% at 7-day follow-up, and the incidence rate (or density) of active CMV infection was $2.75 \%$ per person-day during the 21-day follow-up [13]. However, in another observational study on critically ill patients with sepsis, CMV reactivation incidence reached $17.9 \%$ [14]. Our finding of the lower incidence of CMV reactivation in patients with immunocompetent critically ill compared with most studies might have been attributable to ethnic differences. The inclusion criteria for studies are more stringent to screen out non-immunosuppressed patients. Also, the detection methods (samples, time points, and monitoring periods) of CMV in different studies and the selection of different diseases as subjects may be related to the incidence of CMV reactivation.

Previous studies had demonstrated that sepsis was associated with the development of CMV reactivation $[4,5,15,16]$, which was consistent with our results. Sepsis can induce CMV reactivation mainly through sepsis-related cytokine storm triggering transcriptional CMV replication, a mechanism that has been confirmed in animal models $[4,17,18]$. Several studies have shown that CMV infection can impair the cardiac and renal function, increasing cardiac and renal biochemical indicators $[19,20]$, which is consistent with this study's results. The reason beyond this association is related to the direct pathological damage caused by $\mathrm{CMV}$ infection and indirect damage caused by inflammatory factors. Recent studies have shown that CMV infection can affect the body's development and metabolic level and induce metabolic syndrome, which manifests as a chronic consumptive condition [21]. It can cause abnormal growth and development of the fetus and decrease lipids, BMI, and hemoglobin in adults [22, 23]. Our study found that the height, weight, and hemoglobin levels of the CMV reactivation group were lower, which may be related to CMV reactivation's metabolic disorder.

Many studies have explored the risk factors for CMV reactivation. A meta-analysis [24] suggested that CMV reactivation was strongly associated with sepsis, mechanical ventilation, and hypertension induced by glucocorticoids and catecholamines. However, there was no correlation for disease scores, such as the APACHE and SOFA scores. Simultaneously, there was no evidence that CMV reactivation was age-related, but whether it was gender-related or not remained inconsistent. Besides, several clinical studies predicted CMV reactivation by cytokine levels (such as IL-6, IL-10, and TNF-a) [11, 14]. However, the current results were mostly negative, and no consistent results were found, which was similar to our study results. The reason may be related to the clinical condition's complexity, and it is difficult to analyze the relationship between the immune system and CMV reactivation. 
We found that $\mathrm{BMI}, \mathrm{Hb}$, and sepsis were independent risk factors for $\mathrm{CMV}$ reactivation through a multivariate logistic regression model. It was further found that BMI and $\mathrm{Hb}$ levels had a moderate value for predicting CMV reactivation. There is no relevant study consistent with our results. These results suggested that nutritional status [22] in critically ill patients might be associated with CMV reactivation, but more patients need to be included for further confirmation.

Most of the findings suggested that CMV reactivation can seriously affect the clinical prognosis of nonimmunosuppressed patients, which is consistent with our findings, including prolonged duration of mechanical ventilation \& ECMO, increased incidence of nosocomially acquired infections, and increased length of hospitalization and mortality, among others. [4-9, 11-15, 24, 25]. The causes of these adverse prognoses are various, including direct injury (such as CMV pneumonia) and indirect injury (such as immune disorder) [4].

Nonetheless, these results must be interpreted with caution and several limitations should be borne in mind. First, this study was a single-center observational study. The number of patients included was relatively insufficient to comprehensively evaluate CMV's epidemiological characteristics with developing countries' (Chinese) critically ill patients. Second, the number of patients with sepsis was insufficient for subgroup analysis. Third, some patients had CMV reactivation on the day of ICU admission, presumably before ICU admission, which might impact outcomes. Fourth, the capacity to incorporate multivariate logistic regression models was low, decreasing in statistical fitness.

\section{Conclusions}

The incidence of $\mathrm{CMV}$ reactivation was $18.3 \%$ on the immunocompetent mechanical ventilation patients. Furthermore, $\mathrm{CMV}$ reactivation prolonged invasive mechanical ventilation, increased hospitalization expenses, prolonged ICU hospitalization, and increased 90-day mortality. CMV reactivation was also associated with higher transfusion and complications rates. $\mathrm{BMI}, \mathrm{Hb}$, and sepsis were independent risk factors for $\mathrm{CMV}$ reactivation. $\mathrm{BMI}$ and $\mathrm{Hb}$ may predict $\mathrm{CMV}$ reactivation.

\section{Abbreviations}

CMV: Cytomegalovirus; ICU: Intensive care unit; ARDS: Acute respiratory distress syndrome; AKI: Acute kidney injury; MV: Mechanical ventilation; IMV: Invasive Mechanical Ventilation; BMl: Body Mass Index; APACHE $₫$ : Acute Physiology and Chronic Health Evaluation; SOFA: Sequential Organ Failure Assessment; P/F: $\mathrm{PaO}_{2} / \mathrm{FiO}_{2}$; QRT-PCR: Quantitative Real-time Polymerase Chain Recation; COPD: Chronic obstructive pulmonary disease; AECOPD: Acute Exacerbation of Chronic Obstructive Pulmonary Disease; $\mathrm{SPO}_{2}$ : Arterial Oxygen Saturation; Th1/Th2: Helper T Lymphocyte 1\&2; PT: Prothrombin Time; APTT: Activated Partial Thromboplastin Time; NT-proBNP: N-terminal Pro-B-type Natriuretic Peptide; AST: Aspartate Aminotransferase; ALT: Alanine Transaminase; T-BIL: Total Bilirubin; Scr: Serum Creatinine; BUN: Serum Urea Nitrogen; Hb: Hemoglobin; Th: T-helper Lymphocytes; Ts: T-suppressor Lymphocytes; NK: Natural Killer; IL: Interleukin; TNF: Tumor Necrosis Factor; INF: Interferon; IQRs: Interquartile Ranges; $\beta$ : Regression 
Coefficient; OR: Odds Ratio; ROC: Receiver Operating Curve; AUC: Area Under Curve; Cl: 95\% Confidence Interval; KM: Kaplan-Meier; HR: Hazard Ratio.

\section{Declarations}

\section{Acknowledgements}

Not applicable.

\section{Authors' contributions}

ZHZ, XSL, LS, XQL, YML conceived and designed the study; ZHZ, ZW, JRZ, YNS collected and aggregated data; ZHZ, XSL, SBC, YHX analyzed the data and wrote the manuscript; XQL, YML, WQH reviewed and revised the manuscript. All authors read and approved the final manuscript.

\section{Funding}

The study was funded by National Science and Technology Major Project (No. 2017ZX10204401), National Natural Science Foundation of China (No. 81970071, 82070084), the Major Special Projects of the Ministry of Science and Technology (No. 2020YFC0841300), Clinical Research and Cultivation Project of Guangzhou Medical University (No. B185004064).

\section{Availability of data and materials}

Data sharing will be considered only on a collaborative basis with the principal investigators, after evaluation of the proposed study protocol and statistical analysis plan.

\section{Ethical approval and consent to participate}

The Ethics Committees of the First Affiliated Hospital of Guangzhou Medical University approved the protocol and consent forms (No. GY-2017-40).

\section{Consent for publication}

Not applicable.

\section{Competing interests}

None of the authors has any conflict of interest to report.

\section{References}

1. Mohamed Zuhair G, Suzanne A, Smit Gabriel, Wallis, et al Estimation of the worldwide seroprevalence of cytomegalovirus: A systematic review and meta-analysis[J]. Rev Med Virol. 2019 May;29(3):e2034. DOl: 10.1002/rmv.2034. 
2. Paul DG. Burden of disease associated with human cytomegalovirus and prospects for elimination by universal immunisation[J]. Lancet Infect Dis. 2012 Oct;12(10): 790-8. DOl: 10.1016/S14733099(12)70197-4.

3. Griffiths Paul, Baraniak Ilona, Reeves Matt. The pathogenesis of human cytomegalovirus. J Pathol[J]. 2015 Jan;235(2):288 - 97. DOI: 10.1002/path.4437.

4. Laurent Papazian Sami, Hraiech Samuel, Lehingue, et al Cytomegalovirus reactivation in ICU patients[J]. Intensive Care Med. 2016 Jan;42(1):28-37. DOl: 10.1007/s00134-015-4066-9.

5. Thomas Marandu Michael, Dombek CharlesH, Cook, et al Impact of cytomegalovirus load on host response to Sepsis[J]. Med Microbiol Immunol. 2019 Aug;208(3-4):295-303. DOI: 10.1007/s00430019-00603-y.

6. Wurzer Paul, Guillory Ashley, Parvizi Daryousch, et al Human herpes viruses in burn patients: A systematic review[J]. Burns. 2017 Feb;43(1):25-33. DOl: 10.1016/j.burns.2016.02.003.

7. David SY, Ong Cristian, Spitoni, Peter MC, Klein Klouwenberg, et al Cytomegalovirus reactivation and mortality in patients with acute respiratory distress syndrome[J]. Intensive Care Med. 2016 Mar;42(3):333-341. DOl: 10.1007/s00134-015-4071-z.

8. Philippe Lachance J, Chen R, Featherstone, et al Association Between Cytomegalovirus Reactivation and Clinical Outcomes in Immunocompetent Critically III Patients: A Systematic Review and MetaAnalysis[J]. Open Forum Infect Dis. 2017 Feb;4(2): ofx029. DOl. 10.1093/ofid/ofx029.

9. Sami Hraiech E, Bonnardel C, Guervilly, et al. Herpes simplex virus and Cytomegalovirus reactivation among severe ARDS patients under veno-venous ECMO[J]. Ann Intensive Care. 2019 Dec;"9(1):142. 10.1186/s13613-019-0616-6. DOI.

10. Roy F, Chemaly Sunwen, Chou Hermann, Einsele, et al Definitions of Resistant and Refractory Cytomegalovirus Infection and Disease in Transplant Recipients for Use in Clinical Trials[J]. Clin Infect Dis. 2019 Apr;68(8):1420-1426. DOI: 10.1093/cid/ciy696.

11. Frantzeska G. Cytomegalovirus reactivation in a general, nonimmunosuppressed intensive care unit population: incidence, risk factors, associations with organ dysfunction, and inflammatory biomarkers[J]. J Crit Care. 2015 Apr;;30(2):276 - 81. 10.1016/j.jcrc.2014.10.002. Fra ntzeskaki, EiriniSofia Karampi, Christina Kottaridi, et al. ( $\therefore$ DOI.

12. Li Xi, Huang Yongbo, Xu Zhiheng, et al Cytomegalovirus infection and outcome in immunocompetent patients in the intensive care unit: a systematic review and meta-analysis[J]. BMC Infect Dis. 2018 Jun;18(1):289. DOI: 10.1186/s12879-018-3195-5.

13. Shah DB, Ekta G, Archana R, et al. Incidence of Active Cytomegalovirus Infection and Its Influence on Outcome Among Non-Immunosuppressed Cirrhotic Adults Requiring Critical Care in Liver-ICU[J]. Open Forum Infectious Diseases. 2019 Jun;Supplement 2:789. DOI: 10.1093/ofid/ofz360.1983.

14. Taylon Felipe, Silva, Virgínia Márcia Concato, Fernanda Tomiotto-Pellissier, et al. Reactivation of Cytomegalovirus Increases Nitric Oxide and IL-10 Levels in Sepsis and is Associated with Changes in Renal Parameters and Worse Clinical Outcome[J]. Sci Rep. 2019 Jun;9(1):9016. DOI: 10.1038/s41598-019-45390-x. 
15. Alexandra Heininger Helene, Haeberle Imma, Fischer, et al Cytomegalovirus reactivation and associated outcome of critically ill patients with severe Sepsis[J]. Crit Care. 2011;15(2):R77. DOI: 10.1186/cc10069.

16. Andre C, Kalil, Diana F, Florescu. Is cytomegalovirus reactivation increasing the mortality of patients with severe Sepsis?[J]. Crit Care. 2011;15(2):138. DOI:10.1186/cc10093.

17. Richard S, Hotchkiss G, Monneret D, Payen. Sepsis-induced immunosuppression: from cellular dysfunctions to immunotherapy[J]. Nat Rev Immunol. 2013 Dec;13(12):862-74. DOI:10.1038/nri3552.

18. Charles H, Cook J, Trgovcich, Peter D, Zimmerman, et al. Lipopolysaccharide, tumor necrosis factor alpha, or interleukin-1 beta triggers reactivation of latent cytomegalovirus in immunocompetent mice[J]. J Virol. 2006 Sep;80(18):9151-8. DOI:10.1128/JVI.00216-06.

19. Haoran Wang Geng, Peng Jing, Bai, et al Cytomegalovirus Infection and Relative Risk of Cardiovascular Disease (Ischemic Heart Disease, Stroke, and Cardiovascular Death): A Meta-Analysis of Prospective Studies Up to 2016[J]. J Am Heart Assoc. 2017 Jul;6(7):e005025. DOI: 10.1161/JAHA.116.005025.

20. Amit D, Raval KristinD, Kistler Yuexin, Tang, et al. Epidemiology, Risk factors and Outcomes Associated with Cytomegalovirus in Adult Kidney Transplant Recipients: A Systematic Literature Review of Real-World Evidence[J]. Transpl Infect Dis. 2020 Oct;e13483. DOI: 10.1111/tid.13483.

21. Karen B, Fowler, Suresh B, Boppana. Congenital cytomegalovirus infection[J]. Semin Perinatol. 2018 Apr;42(3):149-154. DOI: 10.1053/j.semperi.2018.02.002.

22. Shannon Fleck-Derderian. William McClellan, Janet M Wojcicki. The association between cytomegalovirus infection, obesity, and metabolic syndrome in U.S. adult females[J]. Obesity (Silver Spring). 2017 Mar;25(3):626-633. DOI: 10.1002/oby.21764.

23. Jerrald L, Rector G, Neil Thomas, Victoria E, Burns, et al Elevated $\mathrm{HbA}(1 \mathrm{c})$ levels and the accumulation of differentiated T cells in CMV(+) individuals[J]. Diabetologia. 2015 Nov;58(11):2596 - 605. DOI: 10.1007/s00125-015-3731-4.

24. Aljamaan AwadAl-Omari,Fadi, Alhazzani Waleed, et al Cytomegalovirus infection in immunocompetent critically ill adults: literature review[J]. Ann Intensive Care. 2016 Dec;6(1):110. DOI: 10.1186/s13613-016-0207-8.

25. Chen Wen-LiangYChin-Ming, Lee Wen-Ying. Ventilator-associated cytomegalovirus organizing pneumonia in an immunocompetent critically ill patient[J]. J Microbiol Immunol Infect. 2017 Feb;50(1):120-122. DOI: 10.1016/j.jmii.2014.11.012.

\section{Figures}


From 30 June 2017 to 01 July 2018

1350 patients were transferred to ICU

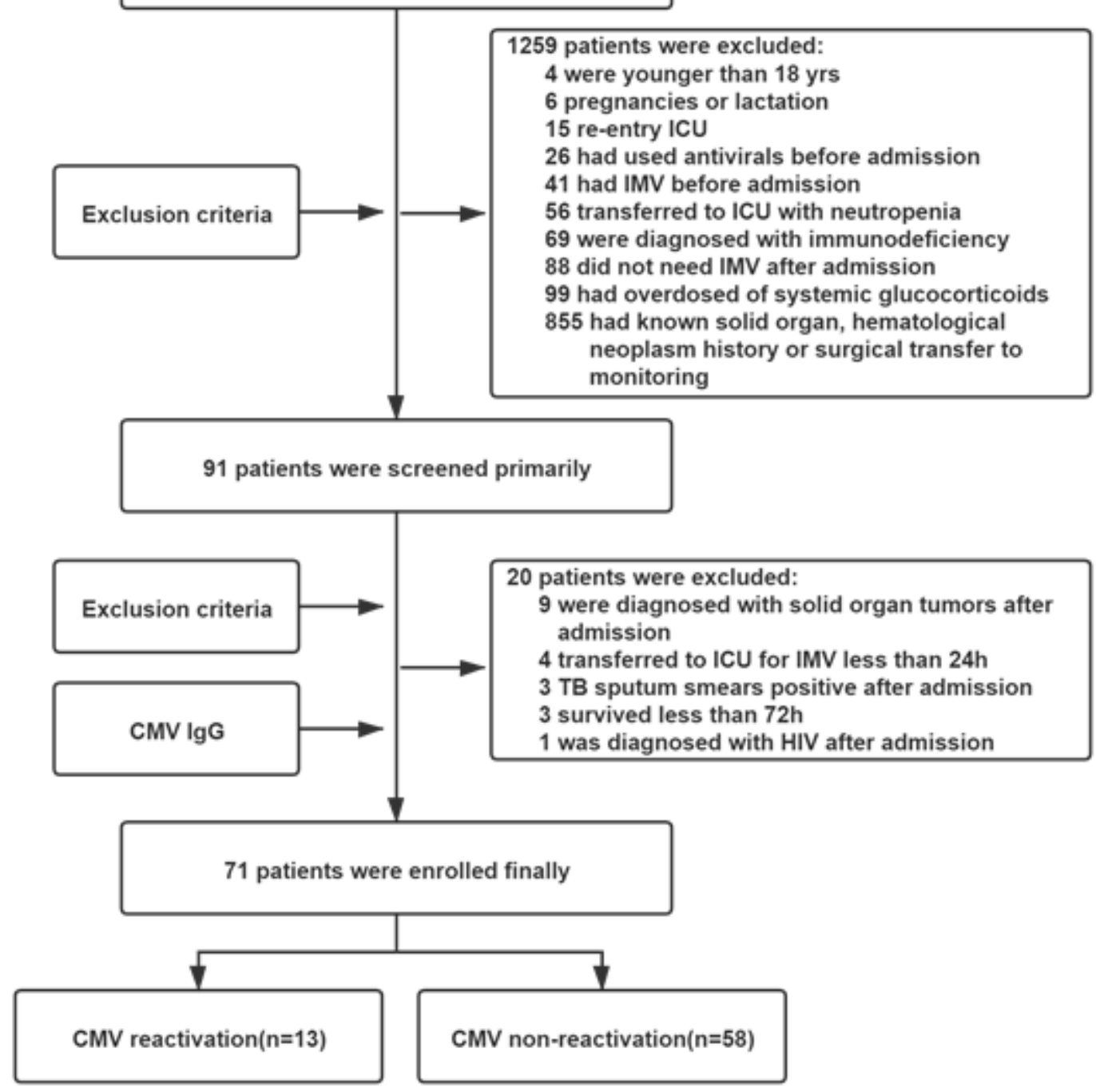

Figure 1

Flowchart of patient enrollment. 


\section{CMV reactivation $(n=13) 18.3 \%$ \\ CMV non-reactivation( $n=58) 81.7 \%$}

\section{$\mathrm{N}=71$}

Figure 2

Incidence of CMV reactivation 28-day at ICU admission. 


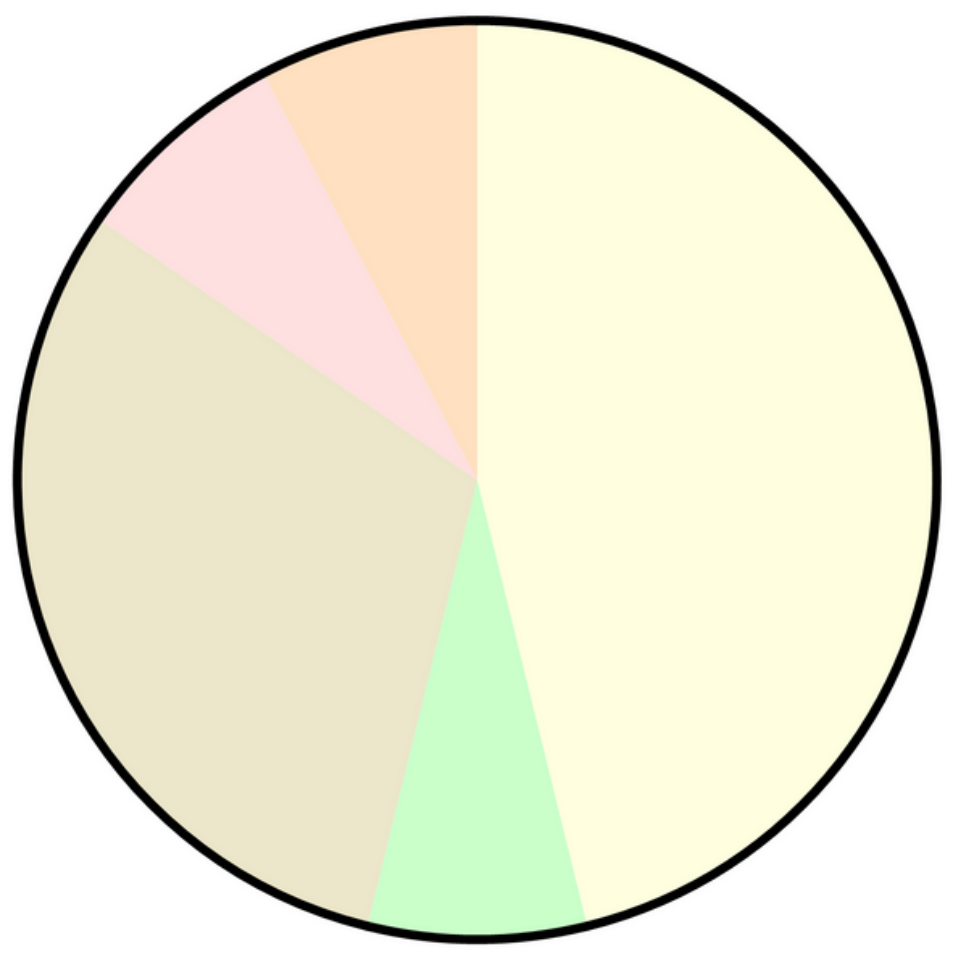

$\mathrm{N}=13$

Figure 3

Time of CMV reactivation at 28-day ICU admission.

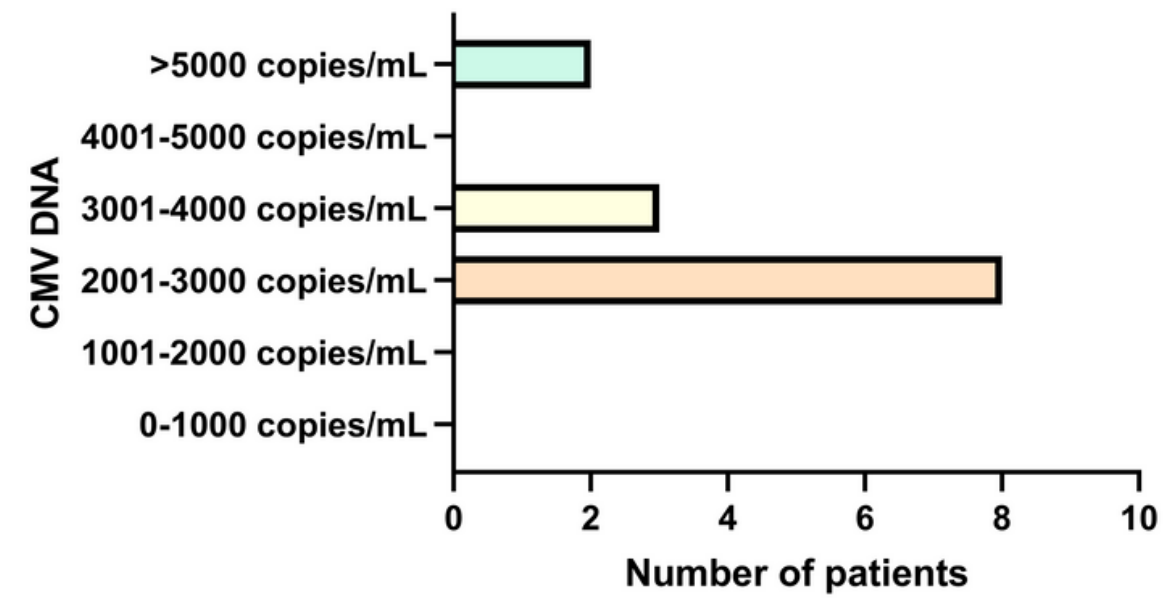

Figure 4

DNAemia of CMV reactivation at 28-day ICU admission. $\square \mathrm{D} 0(\mathrm{n}=6) 46.1 \%$

$\square \mathrm{D} 7(\mathrm{n}=1) 7.7 \%$

$\square \mathrm{D} 14(\mathrm{n}=4) 30.8 \%$

$\square \mathrm{D} 21(\mathrm{n}=1) 7.7 \%$

$\square$ D28(n=1) $7.7 \%$
ㅁ $61.5 \%(\mathrm{n}=8) 2001-3000 \mathrm{copies} / \mathrm{mL}$

口 23.1\%(n=3) 3001-4000 copies $/ \mathrm{mL}$

ㅁ $15.4 \%(\mathrm{n}=2)>5000$ copies $/ \mathrm{mL}$ 
ROC of BMI and $\mathrm{Hb}$

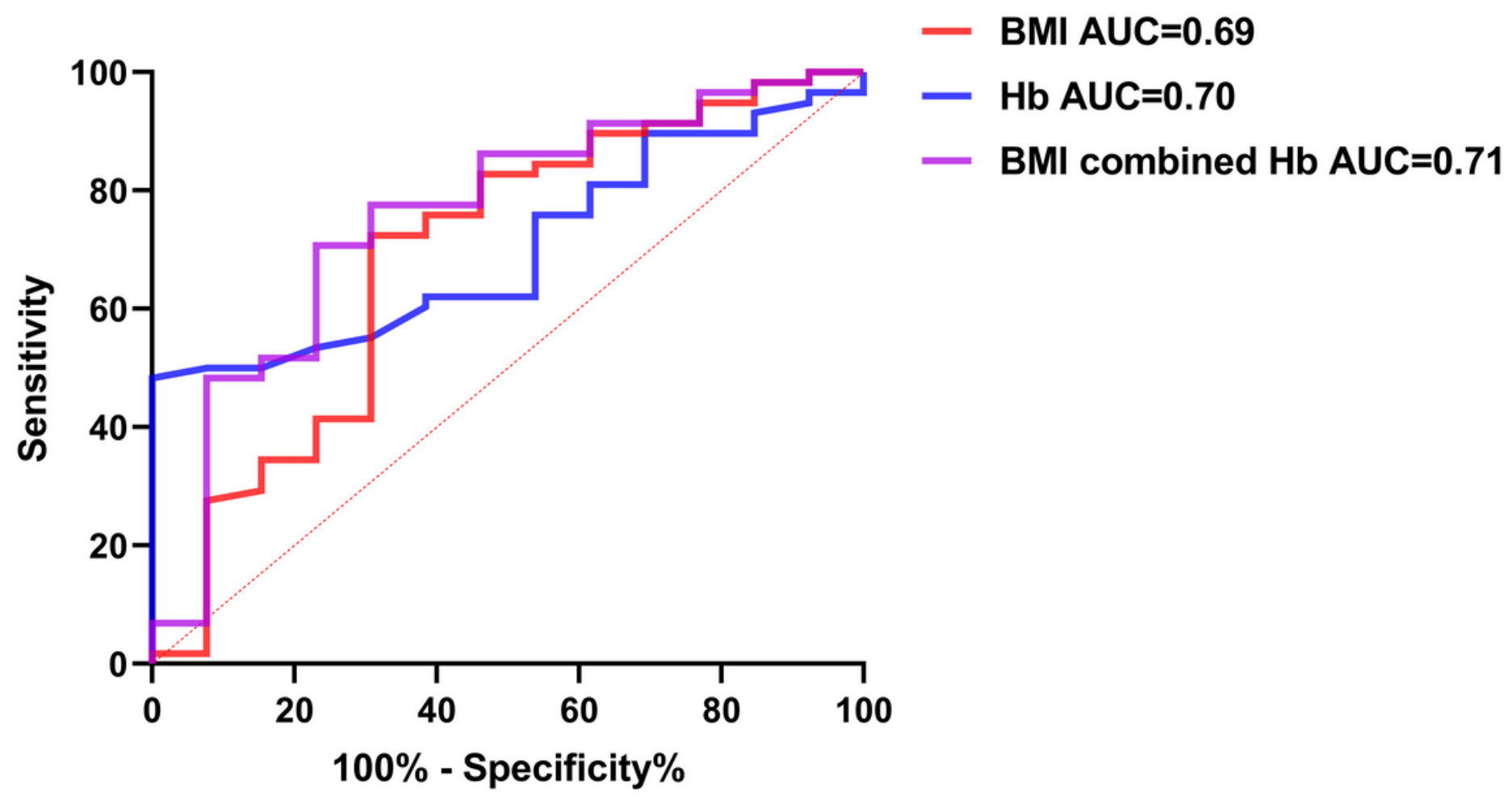

Figure 5

$\mathrm{ROC}$ curve evaluation of the predictive value of $\mathrm{BMI}$ and $\mathrm{Hb}$ for $\mathrm{CMV}$ reactivation.

Survival of 90-day after entry ICU

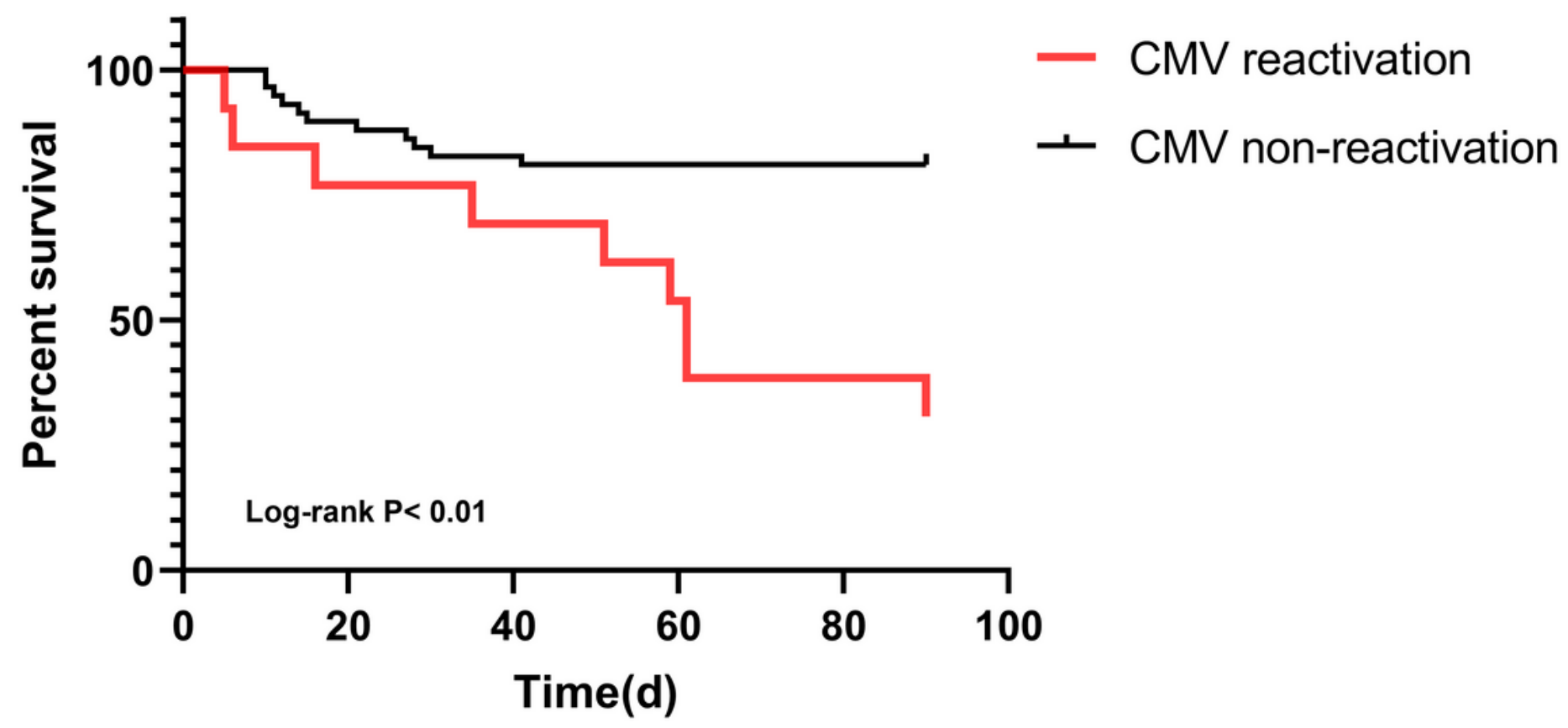


Figure 6

Kaplan-Meier curves to assess the effect of CMV reactivation on 90-day survival.

\section{Supplementary Files}

This is a list of supplementary files associated with this preprint. Click to download.

- STROBEchecklistcasecontrol.pdf 